\title{
Médiévales
}

Langues, Textes, Histoire

67 | automne 2014

Histoires de Bohême

\section{Arthur et ses barons rebelles. La fin remaniée et abrégée de la Suite Vulgate du Merlin dans le manuscrit du cycle du Graal (Paris, BnF, fr. 344, ca 1295)}

Arthur and his Rebel Barons. The Abridgment and Reworking of the End of the Vulgate Sequel to Merlin in the Lancelot-Grail Manuscript (Paris, BnF, fr. 344, ca 1295)

Irène Fabry-Tehranchi

\section{(2) OpenEdition}

Journals

Édition électronique

URL : https://journals.openedition.org/medievales/7429

DOI : $10.4000 /$ medievales.7429

ISSN : 1777-5892

Éditeur

Presses universitaires de Vincennes

Édition imprimée

Date de publication : 31 décembre 2014

Pagination : 121-142

ISBN : 978-2-84292-422-5

ISSN : 0751-2708

Référence électronique

Irène Fabry-Tehranchi, « Arthur et ses barons rebelles. La fin remaniée et abrégée de la Suite Vulgate du Merlin dans le manuscrit du cycle du Graal (Paris, BnF, fr. 344, ca 1295) », Médiévales [En ligne], 67 | automne 2014, mis en ligne le 31 décembre 2016, consulté le 22 avril 2022. URL : http:// journals.openedition.org/medievales/7429; DOI : https://doi.org/10.4000/medievales.7429 


\section{Irène Fabry-Tehranchi}

\section{Arthur et ses barons rebelles \\ La fin remaniée et abrégée de la Suite Vulgate du Merlin dans le manuscrit du cycle du Graal (Paris, BnF, fr. 344, ca1295)}

On conserve plus d'une trentaine de manuscrits comprenant le Merlin et la Suite Vulgate. Ces ouvrages, réalisés entre le deuxième quart du XIII ${ }^{\mathrm{e}}$ siècle et la fin $\mathrm{du} \mathrm{XV}^{\mathrm{e}}$ siècle, nous renseignent sur le succès de ces textes et l'importance de leur diffusion tout au long du Moyen Âge. Le Merlin et sa suite ont été intégrés au cycle du Graal dès la première moitié $\mathrm{du} \mathrm{XIII}^{\mathrm{e}}$ siècle. Les manuscrits cycliques complets, dont il subsiste une dizaine d'exemplaires ${ }^{1}$, sont cependant une minorité par rapport à ceux qui transmettent de façon distincte un ou plusieurs des textes de la Vulgate arthurienne.

Le manuscrit Paris, BnF, fr.344, qui contient l'intégralité du cycle du Graal (même s'il comprend quelques lacunes à la fin de la Queste ainsi qu'au début et à la fin de la Mort Artu), présente un intérêt textuel et iconographique particulier en ce qui concerne la conclusion de la Suite Vulgate $^{2}$. Cet ouvrage a été produit dans la région de Metz-Verdun vers

1. Il s'agit de: Paris, BnF, fr.747-751 (reconstitué à partir de volumes actuellement distincts); Bonn, Universitäts- und Landesbibliothek, 526; Paris, BnF, fr.344 et fr. 110; exAmsterdam, BPH, 1-Oxford, Bodleian Library, Douce 215-Manchester, Rylands Library, fr. 1 (omettant la Suite Vulgate); Londres, British Library, Add. 10292-94; Paris, Arsenal, 3479-80; BnF, fr. 117-120, fr.98 et fr. 113-116. Deux manuscrits du Xve siècle (Paris, Arsenal, 3350 et New York, Pierpont Morgan Library, 38) offrent une version abrégée du cycle du Graal.Tous les manuscrits cycliques présentent la version courte $(\beta)$ du Merlin et de la Suite Vulgate. Sur la tradition manuscrite de ces textes, voir A. Micha, «Les manuscrits du Merlin en prose de Robert de Boron», Romania, 79 (1958), p. $78-94$ et 145-174; R. TRAChSLER, «Merlin chez Jules César. De l'épisode de Grisandole à la tradition manuscrite de la Suite $d u$ Merlin», Studi Francesi, 133 (2001), p. 61-71; ID., «Pour une nouvelle édition de la SuiteVulgatedu Merlin », Vox Romanica, 60 (2001), p. 128-148.

2. Toutes les miniatures de ce manuscrit seront reproduites et commentées dans l'ouvrage que je prépare en collaboration avec C. Nicolas, L'Iconographie du LancelotGraal, Turnhout, 2015 [à paraître]. 
1290-1300 et il est écrit dans un dialecte lorrain ${ }^{3}$. Dans ce recueil cyclique formé de sénions ou cahiers de six bifeuillets, on passe d'une œuvre à l'autre au sein d'un même cahier et sur le même folio. La continuité cyclique prime donc sur l'autonomie codicologique de chaque partie, même si l'illustration marque différemment le seuil de chaque texte. Le Merlin et sa suite se situent, dans la chronologie narrative, après l'Estoire del saint Graal et avant le Lancelot. Dans le fr.344, la fin de la Suite Vulgate et le commencement du Lancelot sont particulièrement mis en valeur, alors que le passage du Lancelot à la Queste del Saint Graal est uniquement indiqué par une initiale historiée compartimentée (fo 186$)$. Ce type d'illustration intervient ponctuellement dans le Lancelot à l'occasion de la mort du roi Ban et de l'enlèvement de son fils par la Dame du Lac.

L'étude de la mise en page et de l'iconographie des manuscrits du Merlin et de sa suite, et plus particulièrement celle des manuscrits du cycle du Graal, éclaire la façon dont les concepteurs de ces ouvrages ont envisagé l'articulation et la hiérarchisation de ses différentes parties. Le programme iconographique du fr.344 témoigne du déséquilibre perceptible dans la majorité des manuscrits entre l'illustration du Merlin et celle de la suite. L'ouvrage comprend en moyenne 65 miniatures pour 100 folios. Dans l'état de conservation actuel, la Suite Vulgate est le texte le plus illustré de la compilation, avec plus d'une miniature par folio ${ }^{4}$. L'irrégularité de cette distribution profite donc à la suite aux dépens du Merlin propre. L'Estoire de Merlin semble pourtant conçue comme un tout car le début du Merlin est mis en valeur par une image frontispice compartimentée, tandis que rien ne distingue la miniature marquant le commencement de la suite.

Dans le fr.344, le Merlin comprend une seule image, hormis la miniature frontispice immédiatement suivie d'une initiale historiée. Avec 95 miniatures, l'illustration de la Suite Vulgate est foisonnante: la guerre, omniprésente, constitue l'arrière-plan de nombreuses scènes de combat, de chevauchée et plus rarement de navigation. Comme l'indiquait déjà Alexandre Micha ${ }^{5}$ :

3. Voir H.-J.Martin et J.Vezin éd., Mise en page et mise en texte du livre manuscrit, Paris, 1990, p. 124-125; A. STONES, «"Mise en page" in the French Lancelot-Grail: The First 150 Years of the Illustrative Tradition», dans C. Dover éd., A Companion to the LancelotGrail Cycle, Cambridge, 2003, p. 132 et 143, fig. 5. Pour A Stones, l'artiste de BnF, fr 344

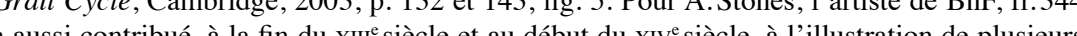
aussi contibué, à fin du $x$ plusieurs utres manus de Cantimpré), qui daterait de 1276, Metz, AD de la Moselle, H 4085 (5) (charte de Sainte Glossinde de Metz, rédigée en 1293), et Wien, ÖNB, Cod. 1814 (Pontifical de Strasbourg), datant de 1311

4. Elle est suivie de la Mort Artu (73 miniatures pour 100 folios), du Lancelot (61 pour 100), de l'Estoire (51 pour 100) et de la Queste (39 pour 100), tandis que dans le Merlin propre le rapport n'est que de 15 pour 100.

5. A. MichA, «Les sources de la Vulgatedu Merlin», dans De la chanson de geste au roman, Genève, 1976, p. 310.
Les chansons de geste ont laissé plus d'une trace dans ce roman qui est, en fin de compte, beaucoup plus une épopée qu'un roman courtois, puisque

l'amour n'y occupe aucune place, alors que les faits d'armes et les tentatives diplomatiques constituent le plus clair de l'action.

Les «Premiers faiz le roy Artu», titre donné à la Suite Vulgate dans le manuscrit du cycle du Graal, Bonn, Universitäts- und Landesbibliothek 256 ( $\mathrm{f}^{\circ} 82$ ), sont donc au cour du texte comme de l'illustration, témoignant ainsi de sa réception. Les scènes de cour (principalement des conseils mais aussi l'envoi et la réception de messagers) sont également liées aux circonstances militaires, politiques ou diplomatiques. L'illustration de ce texte dans le fr.344 met en avant les événements qui marquent l'histoire des rois de Bretagne et plus particulièrement le règne d'Arthur, dans sa lutte contre les barons rebelles et contre les Saxons. L'iconographie et le remaniement textuel de la fin de la Suite Vulgate et du début du Lancelot dans le fr. 344 sont intéressants car ils nous renseignent sur une façon particulière de concevoir la royauté et les relations féodales.

Ce manuscrit présente une version textuelle abrégée unique de la fin de la Suite Vulgate qui commence à la fin du fo $182^{6}$. Elle suit la soumission de Loth à Arthur, le tournoi de la Table Ronde et l'envoi de Loth et de ses fils en ambassade auprès des barons rebelles (Pl. I, p. 1381) ${ }^{7}$, peu après le passage où se greffe le Livre d'Artus dans le manuscrit BnF, fr. $337^{8}$. Les rois de Bénoïc et de Gaunes, alliés d'Arthur, rentrent sur le continent pour affronter Claudas. Loth et ses fils négocient pour que trois des rois révoltés contre Arthur se rendent à Logres, où ils sont reçus en grande pompe. À cette occasion ont lieu plusieurs adoubements et un tournoi est organisé. Les rois chrétiens décident alors, sur le conseil de Loth et de Gauvain, de rendre hommage à Arthur. Cette réconciliation constitue, dans le fr.344, le point majeur de la fin du texte

Nous étudierons les choix narratifs, poétiques et idéologiques qui président à l'abrègement de la fin de la Suite Vulgate dans le fr. 344 en mettant en perspective la spécificité de sa clôture iconographique. Dans ce manuscrit, la relation entre Arthur et ses vassaux se trouve en

6. Une transcription du passage remanié figure dans I.FabRY-Tehranchi, Texte $e$ images des manuscrits du Merlin et de la Suite Vulgate: mise en cycle et poétique de la continuation, ou suite et fin d'un roman de Merlin?, Thèse, Paris III, dir. Michelle Szkilnik, 2011, Annexe 6.

7. Le Livre du Graal, I. Joseph d'Arimathie, Merlin, Les premiers faits du roi Arthur, dir. D. Poirion et P. Walter, éd.A.Berthelot, R. Deschaux, I. Freire-Nunes et G. Gros, Paris, 2001 [cité dorénavant Pl. I].

8. Il s'agit d'une autre suite du Merlin qui succède à la Suite Vulgate dans un manuscrit unique du Nord de la France datant des années 1230-1240: voir The Vulgate Version of the Arthurian Romances, t. VII, Supplement : Le Livre d'Artus, with Glossary and Index of Names and Places, éd. H. O. SOMMER, Washington, 1916. 
effet sensiblement modifiée. Nous examinerons les implications de ce remaniement et de cet abrègement dans le cadre de la formation d'un ensemble romanesque comprenant l'intégralité du cycle du Graal.

\section{Abrègement et mise entre parenthèse des aventures merveilleuses, chevaleresques et courtoises de la fin de la Suite Vulgate}

Le remaniement de la fin de la Suite Vulgate met entre parenthèses les épisodes plus romanesques de la version commune et renforce la cohérence épique de l'œuvre autour de la figure d'Arthur, souverain légitime et habile chef de guerre, confronté à la dissidence de ses barons. L'abrègement final porte en effet sur un enchaînement d'épisodes amoureux ou chevaleresques parfois teintés de merveilleux, dont le développement est pourtant plus caractéristique des romans arthuriens que la succession de conflits militaires ou les questions d'ordre féodal. La vie de Merlin et la fin du prophète ne sont plus au centre du texte éponyme et de sa continuation.

Le fr.344 omet ainsi quatre des six rencontres entre Merlin et son amie $^{9}$, les deux dernières évoquant l'oreiller enchanté dont se sert Viviane pour endormir Merlin puis l'enserrement de ce dernier. Ce raccourcissement tend peut-être à éviter des effets de répétition, voire des discordances entre la suite du Merlin et les autres textes du cycle. Dans la version longue du Lancelot, l'évocation de l'enlèvement de Lancelot par la dame du Lac est l'occasion de noircir le portrait de l'enchanteur, enfermé par son amante dans une fosse au sein de la forêt de Darnantes ${ }^{10}$, alors que la Suite Vulgate propose une version plus nuancée de sa relation avec Viviane et de son enserrement. L'abrègement final et l'absence des amours de Merlin et Viviane favorisent le passage d'un texte à l'autre puisque la figure de Merlin s'efface dans le Lancelot $^{11}$.

La disparition du prophète enserré par Viviane dans une prison invisible constitue un défi sur le plan iconographique. Elle n'est illustrée que dans trois manuscrits de la Suite Vulgate datant de la fin du XIII ${ }^{\mathrm{e}}$ siècle $^{12}$. La représentation de Merlin et Viviane au début du Lancelot est encore

9. Voir Pl. I, p. 1452, 1525-1526, 1559-1560 et 1630-1632.

10. Ce passage fait l'objet de variations textuelles. Pour la version longue, voir Lancelot: roman en prose du XIII siècle, éd. A. Мıсна, Genève, 1980, t. 7, § VIa-10 et, pour la version courte, Le Livre du Graal. II Lancelot, p. 41-46.

11. Voir F.Dubost, Aspects fantastiques de la littérature narrative médiévale: XIIXIII' siècles. L'autre, l'ailleurs, l'autrefois, Paris, 1991, t. II, p. 743-745; A. ComBEs, Les Voies de l'aventure : réécriture et composition romanesques dans le Lancelot en prose, Paris, 2001, p. 65-66; N. KoвLE, Les Prophéties de Merlin en prose : le roman arthurien en éclats, Paris, 2009 , p. $250-256$

12. Bonn, ULB, 526, fo 166 ; Paris, BnF, fr. $110, \mathrm{f}^{\circ} 159 \mathrm{v}^{\circ} ; \mathrm{BnF}$, fr. 749 , fo 331 Voir I.FABRY, «Continuity and Discontinuity: Illuminating and Interlacing the plus rare: les deux personnages sont représentés en discussion dans deux manuscrits: le fr. $344, \mathrm{f}^{\circ} 187 \mathrm{v}^{\circ}$ (Figure 1) ${ }^{13}$, et le New York, Pierpont Morgan Library, $805, \mathrm{f}^{\circ} 10$, où le teint foncé de l'enchanteur trahit ses origines diaboliques ${ }^{14}$. La miniature du fr. 344 met en scène la conversation des deux personnages et l'autorité du discours de Merlin. Elle insiste moins sur la lubricité du personnage ou la violence de sa disparition que sur la relation pédagogique qu'il entretient avec son amie. L'absence de figuration des rencontres entre Merlin et Viviane dans la Suite Vulgate du fr. 344 gomme aussi la construction progressive de leur relation amoureuse, tandis que l'abrègement du texte réduit le caractère sériel de leurs rencontres Ces choix textuels et iconographiques, qui s'inscrivent dans la perspective d'une plus grande cohérence cyclique, privilégient le Lancelot et atténuent la dimension courtoise de l'histoire de Merlin et Viviane.

Plusieurs aventures de la fin de la Suite Vulgate permettaient en outre d'introduire des épisodes chevaleresques à caractère merveilleux, selon un modèle narratif qui se développe dans le Lancelot et la Queste. Or, la fin du fr.344 se caractérise par l'absence de la quête de Merlin et la disparition

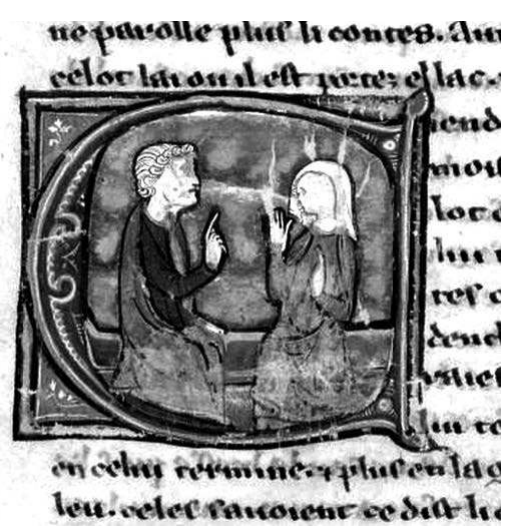

Figure 1. Lancelot: Merlin et Viviane. Paris, BnF, fr. $344, \mathrm{f}^{\circ} 187 \mathrm{v}^{\circ} \mathrm{b}$.

Adventures of Viviane and Merlin in the Prose Merlin», Marginalia, 3 (2006) http://www.marginalia.co.uk/journal/06illumination/fabry.php (01/04/2012)

13. Dans le fr.344, qui supprime la narration de l'enserrement du prophète à la fin de la Suite Vulgate, le placement d'une initiale historiée mettant en scène Merlin et Viviane au début du Lancelot souligne la primauté donnée à la version de l'épisode tel qu'il est raconté par Viviane elle-même. Merlin ne finit plus dans une prison amoureuse où il jouit exclusiver Vivich do cou gate. Dan le Lancelot, il est une créature démoniaque et luxurieuse dont se débrasse définitivement Viviane, anxieuse de préserver sa virginité

14. Voir M.MeuWESE, «Inaccurate Instructions and Incorrect Interpretations: Errors and Deliberate Discrepancies in Illustrated Prose Lancelot Manuscripts», Bulletin bibliographique de la Société internationale arthurienne, 54 (2002), p. 223-224. 
des aventures d'Enadain, le chevalier nain, et de son amie Bianne ${ }^{15}$. Le fr.344 passe également sous silence l'engendrement d'Hector par le roi Ban et la fille du seigneur des Marais, alors que le jeune homme est amené à jouer un rôle relativement important dans les aventures entrelacées du Lancelot. Cette union qui s'opère à l'initiative de Merlin rappelle la conception d'Arthur et évoque celle de Galaad, mais elle ne fait l'objet d'une représentation que dans un seul manuscrit, le New Haven, Beinecke Library, 227, fo $292 v^{\circ}$. L'abrègement de la fin de la Suite Vulgate dans fr. 344 suscite alors l'omission d'éléments à caractère cyclique.

La continuation ne se structure donc plus autour de la biographie de Merlin. Le remaniement gomme certaines contradictions narratives liées à la construction cyclique, mais entraîne aussi paradoxalement la suppression d'épisodes dont la composition et la tonalité pouvaient au contraire renforcer l'intégration du versant historico-épique incarné par le Merlin et sa suite et des œuvres du cycle qui mettent davantage l'accent sur les aventures amoureuses et chevaleresques. On privilégie alors l'orientation à la fois politique et épique de la continuation, une veine présente au début du Lancelot, au détriment des éléments romanesques qui y sont ensuite développés.

\section{Remaniement et insistance textuelle et visuelle} sur des questions politiques, féodales et vassaliques

Le remaniement de la fin de la Suite Vulgate dans le fr. 344 se focalise sur les épisodes guerriers et diplomatiques de la fin du texte. De la fin du $\mathrm{f}^{\circ} 182 \mathrm{au}^{\circ} \mathrm{f}^{\circ} 184 \mathrm{v}^{\circ}$ sont évoqués le départ des rois Ban et Bohort pour le continent, l'ambassade du roi Loth et de ses fils, et la lutte contre les Saxons de la coalition chrétienne rassemblée à Logres pour la fête de la sainte Croix. L'abrègement témoigne donc d'une forte inflexion idéologique, exaltant la souveraineté d'Arthur en insistant sur le ralliement puis la contrition et la soumission de ses vassaux.

Hormis sa miniature frontispice initiale, le fr. 344 consacre une seule image à l'illustration du Merlin propre. L'initiale historiée du folio 86 (Figure 2) représente le châtiment des assassins du roi Maine, qui éliminent ce souverain faible pour faire monter sur le trône de Bretagne le puissant baron Vertigier. Au lieu de récompenser ses complices, Vertigier les désavoue

15. L'histoire d'Enadain et les mésaventures de Gauvain font pourtant l'objet d'un traitement iconographique relativement développé dans d'autres ouvrages: de quatre à six miniatures y sont consacrées dans plusieurs manuscrits de la fin du XIII siècle (Oxford, Bodleian Library, Douce 178; Bonn, ULB, 526; Paris, BnF, fr.770, fr.95 et fr. 749) et du début ou milieu du XIv ${ }^{\mathrm{e}}$ siècle (London, British Library, Add.10292; Paris, Arsenal, 3482; New Haven, Beinecke Library, 227). et les condamne à une mort horrible: ils seront traînés par des chevaux ${ }^{16}$. Ce choix iconographique oriente de façon dramatique la lecture du texte et de sa suite. Il interroge la transmission du pouvoir au sein de la lignée des rois de Bretagne et son usurpation. La miniature souligne le caractère central de la réflexion sur la figure royale et sur la relation entre le roi et son entourage. La violence judiciaire exercée par Vertigier a un caractère à la fois exemplaire et prémonitoire: elle s'exerce sur les intermédiaires avant de rattraper son auteur. L'usurpateur meurt consumé dans sa forteresse lors du débarquement d'Uter et Pandragon en Grande Bretagne. Dans la Suite Vulgate, la guerre menée par Arthur permet de conjurer l'invasion saxonne en expulsant définitivement ces ennemis du territoire breton. Le jeune roi est assisté par Merlin, dont l'intervention récurrente contribue à la cohésion de l'œuvre. La construction politique et militaire du royaume, mise en avant dans le programme iconographique, devient un fil directeur du Merlin et de sa suite, créant des jeux de correspondances accentués dans la fin abrégée du fr. 344 .

La dernière enluminure de la Suite Vulgate, dans le fr. 344 , fo $184 \mathrm{a}$ (Figure 3), est une miniature compartimentée, un format fréquent en position frontispice, mais inhabituel pour l'illustration de la fin d'une œuvre. Elle constitue un cas unique dans la tradition iconographique de l'Estoire de Merlin. De taille équivalente à celle qui ouvre le Lancelot, cette miniature propose une synthèse iconographique des événements évoqués

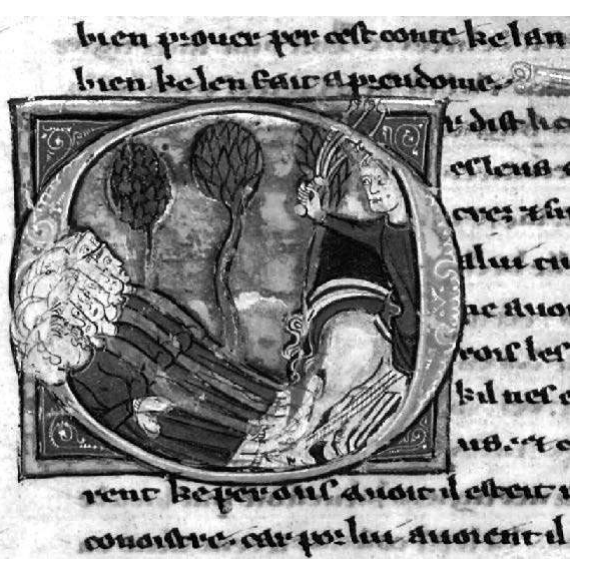

Figure 2. Merlin: Châtiment des assassins du roi Maine. Paris, BnF, fr. $344, f^{\circ} 86$

16. Historiquement, on préférait la trainée des personnages de haut rang, moins humiliante socialement que la pendaison, même si dans les faits les deux châtiments étaient administrés de façon successive. Cette exécution spectaculaire est assez proche de l'écartèlement, un supplice infligé aux traitres et employé en France pour exécuter les régicides. Voir B. MoreL, Une iconographie de la répression judiciaire : le châtiment dans l'enluminure en France du XIII ${ }^{e}$ au $X V^{e}$ siècle, Paris, 2007, p. 115 sq. 
et attire l'attention sur le remaniement de la fin du texte ${ }^{17}$. Quatre des six compartiments de la miniature finale de la Suite Vulgate se rapportent aux relations entre Arthur et les rois révoltés. Trois d'entre eux jouent un rôle de liaison, montrant l'arrivée des rois à Logres puis leur départ et leur retour dans leurs pays. La scène centrale correspond à l'hommage rendu par les barons rebelles à Arthur après la victoire remportée par les Chrétiens sur les Saxons. Elle coïncide avec les dernières phrases du texte, juste avant la formule de transition qui précède l'enluminure (fr. $\left.344, \mathrm{f}^{\circ} 184 \mathrm{a}\right)$ :

.III. jors aprés la baitaille [de Clarence], manderent li princes lou roi Lot et monsignor Gauvain son fil et tindrent grant parlemant antr'ous, tant c'a la fin s'otroierent, per lou consoil lou roi Lot et monsignor Gauvain, qu'il tanroient lor terres del roi Artu. «Or an venez donc, fait messires Gauvains, devant mon signor, et resevez vos terres de lui». Lors s'an vindrent li princes devant lou roi Artu et s'angenoillerent devant lui et li crierent merci de sou qu'il avoient onques estei contre lui. Et li rois lor pardone molt volantiers et lor randit lor terres et il an devindrent si homes et per as fut il puez asasiez et alevez sur toz les princes del monde a son tans.

Non seulement les princes remettent solennellement leurs terres aux mains d'Arthur, mais ils lui demandent pardon pour leur révolte. Le roi, qui fait l'objet d'un sacre, est «responsable de la paix, cette projection sur notre monde imparfait de l'ordre qui règne en haut, de la loi ${ }^{18} \gg$. Comme le souligne Dominique Boutet, le souverain doit faire preuve de miséricorde ${ }^{19}$ :

Le roi doit savoir s'abstraire de la logique féodale comme de celle de la vengeance. La vraie paix en effet, n'est pas celle qui résulterait de l'écrasement du rebelle; elle ne saurait être non plus le fruit d'un compromis dicté par les circonstances et accepté bon gré mal gré par les parties concernées. [...] La seule solution est de rendre une justice authentique.

L'agenouillement exprime l'abaissement et la contrition des barons rebelles mais aussi la reconnaissance de la suzeraineté d'Arthur. L'hommage comprend ainsi deux éléments: l'immixito manuum, où «le vassal, généralement agenouillé, tête nue et sans armes, devant son seigneur, place ses mains jointes dans celles du seigneur qui referme celles-ci sur

17. Dans les autres manuscrits, après la victoire d'Arthur et des barons révoltés contre les Saxons lors de la bataille de Salesbières, la fin de la Suite Vulgate évoque les aventures du chevalier nain Enadain et la quête de Merlin. La voix du prophète se manifeste une dernière fois à Gauvain. Enfin, les rois Ban et Bohort rentrent dans leurs royaumes sur le continent où ils donnent naissance à Lancelot, Bohort et Lionel.

18. G. Duby, Les Trois Ordres ou l'Imaginaire du féodalisme, Paris, 1978, p. 64

19. D. Boutet, Charlemagne et Arthur ou le Roi imaginaire, Paris, 1992, p. 186 elles », c'est la partie du rituel qui est représentée dans la miniature, et le volo, «déclaration de volonté ${ }^{20}$. Dans la miniature, Arthur, bien qu'assis sur son trône, domine ses vassaux de toute sa hauteur. La reconstruction de l'unité politique du royaume autour de la figure du souverain, une des questions fondamentales à laquelle la Suite Vulgate s'efforce de trouver une résolution, devient un élément clef de la fin du texte dans le fr.344.

Dans la version commune de la Suite Vulgate, la réconciliation d'Arthur et des barons rebelles est plus tardive et plus difficile. Avant la bataille de Salesbières, les barons acceptent la coalition contre les Saxons, mais dans un premier temps, cela n'implique pas la reconnaissance de la suzeraineté d'Arthur (Pl. I, p. 1476) :

«Et si volons bien que vous saciés que nous ne somes pas vostre homene onques a nul jour ne tenismes riens de vous. Ains i sommes venu proprement pour l'amour de Jhesu Crist pour la Sainte Eglyse garantir et pour les Saisnes confondre. »

Merlin propose une réconciliation entre le roi et les barons auxquels il recommande de reconnaître Arthur comme leur seigneur: «Et bone chose seroit que vous feïssiés pais a mon signour le roi Artu qui vostres sires devroit estre, si en seriés plus douté et cremu» (Pl. I, p. 1477). Or ces derniers, suivant l'impulsion du roi Urien, s'y refusent. C'est seulement après les premiers affrontements, mais avant la bataille de Clarence, qu'ils acceptent sous serment d'obéir à Merlin, qui leur promet la victoire. Merlin les convainc de faire la paix avec Arthur, ce qui implique de lui prêter hommage (Pl. I, p. 1505-06):

Et [Merlin] lor dist que par son conseil [...] en auront la victoire hui en cel jour. Et cil li dient qu'il en sont prest et apareillié. «Je voel, fait Merlins, que vous me le creantés que vous del tout a mon voloir en ferés». Et cil dient que si feront il molt volentiers. [...] «Ceste gent ne sera sevree ne chacie, devant que vous aurés fait pais au roi Artu. Et c'est ce que vous m'avés acreanté». Quant li barons l'entendent si en ot de tels a qui il ne fu mie bel. Mais autrement ne pooit estre, ains otroient tout a la volenté de Merlin. Et firent tout homage au roi Artu l'un aprés l'autre et rechurent lor terres et lor fiés de lui tout cil qui le durent faire.

20. L'osculum, ou baiser de paix, peut constituer un élément supplémentaire du rite, soulignant le caractère personnel et même physique de la relation entre le vassal et le seigneur. Par la suite, le vassal jure sur des reliques foi et sûreté (fidem et securitatem) à l'égard de son seigneur, ce qui confère un aspect religieux à la cérémonie. Le seigneur l'investit alors de son fief. Voir F. L. GaNSHOF, Qu'est-ce que la féodalité?, Neuchâtel, 1947, p. 92-93; J.-F. Lemarignier, La France médiévale: institutions et société, Paris, 1970, p. 127-128 et 
Le stratagème inventé par Merlin n'est pas nécessaire dans le remaniement de fr.344, où Loth et Gauvain parviennent, aisément et du premier coup, à convaincre les barons. Cette version omet le refus initial des barons et accentue leur humiliation à travers le rituel par lequel ils reconnaissent leur culpabilité et demandent le pardon d'Arthur. L'insistance sur l'abandon de leurs terres et leur restitution symbolique par leur suzerain souligne l'importance du passage et son inscription dans un système féodovassalique qui donne la primauté à la figure royale ${ }^{21}$ :

La perspective politique est celle de l'intérêt général, qui présuppose, aux yeux des auteurs, l'existence d'un ordre dans lequel le roi couronne un système féodal de structure pyramidale. [...] Dans la société féodale ainsi conçue, le monde aristocratique passe après le roi.

La conclusion proposée par le fr.344 consacre la supériorité du roi sur les riches barons, qui acceptent enfin de se reconnaître comme ses vassaux.

Dans le Livre d'Artus, les barons rebelles refusent d'abord les propositions de Merlin qui les encourage à faire hommage à Arthur, et c'est seulement une fois qu'ils sont acculés par les Saxons devant Clarence qu'ils acceptent son invitation. Or la résistance du roi Urien, outré du fait que sa ville a été donnée en fief à Galeschin, le fils du roi Nantres, apporte immédiatement une ombre à cette réconciliation: «Si li firent homage fors solement li rois Yriens qui vint a Galeschin et li contredist sa vile ${ }^{22}$.»

Dans le fr. 344 , la Suite Vulgate se conclut sur une vision pacifiée du royaume de Grande Bretagne qui met en avant la royauté d'Arthur, fondée à la fois sur sa souveraineté, établie au début du texte lors du sacre et du couronnement, et sur sa suzeraineté, reconnue par les barons rebelles à la fin de l'œuvre.

Après le départ des barons, c'est un aspect antérieur de l'action qui semble illustré dans les deux derniers compartiments, au niveau inférieur de la miniature du fr. $344, f^{\circ} 184$ a (Figure 3 ). On peut y voir la navigation de deux figures royales et le débarquement de leurs chevaux. Il s'agit sans doute des rois Ban et Bohort qui, ayant pris congé d'Arthur avant l'arrivée des barons bretons, se rendent sur le continent pour faire face à l'invasion de Claudas. Ban et Bohort jouent un rôle fondamental dans la continuation du Merlin car ils reconnaissent l'autorité d'Arthur quand ce dernier fait face à la rébellion de ses vassaux ${ }^{23}$. Ainsi, au début de la Suite Vulgate, Ban et

21. D. Boutet, Charlemagne et Artur ou le Roi imaginaire..., p. 142.

22. Le Roman de Merlin or The Early History of King Arthur, t. VII, Supplement: Le Livre d'Artus..., p. 25.

23. «Passé le milieu du XII siècle, [le roi] a exploité le principe même de la hiérarchie féodale au profit de son autorité, et de la suzeraineté, a glissé à la souveraineté.» Il est alors présenté «comme placé à un degré suprême qui surplombe, et tous les membres de la

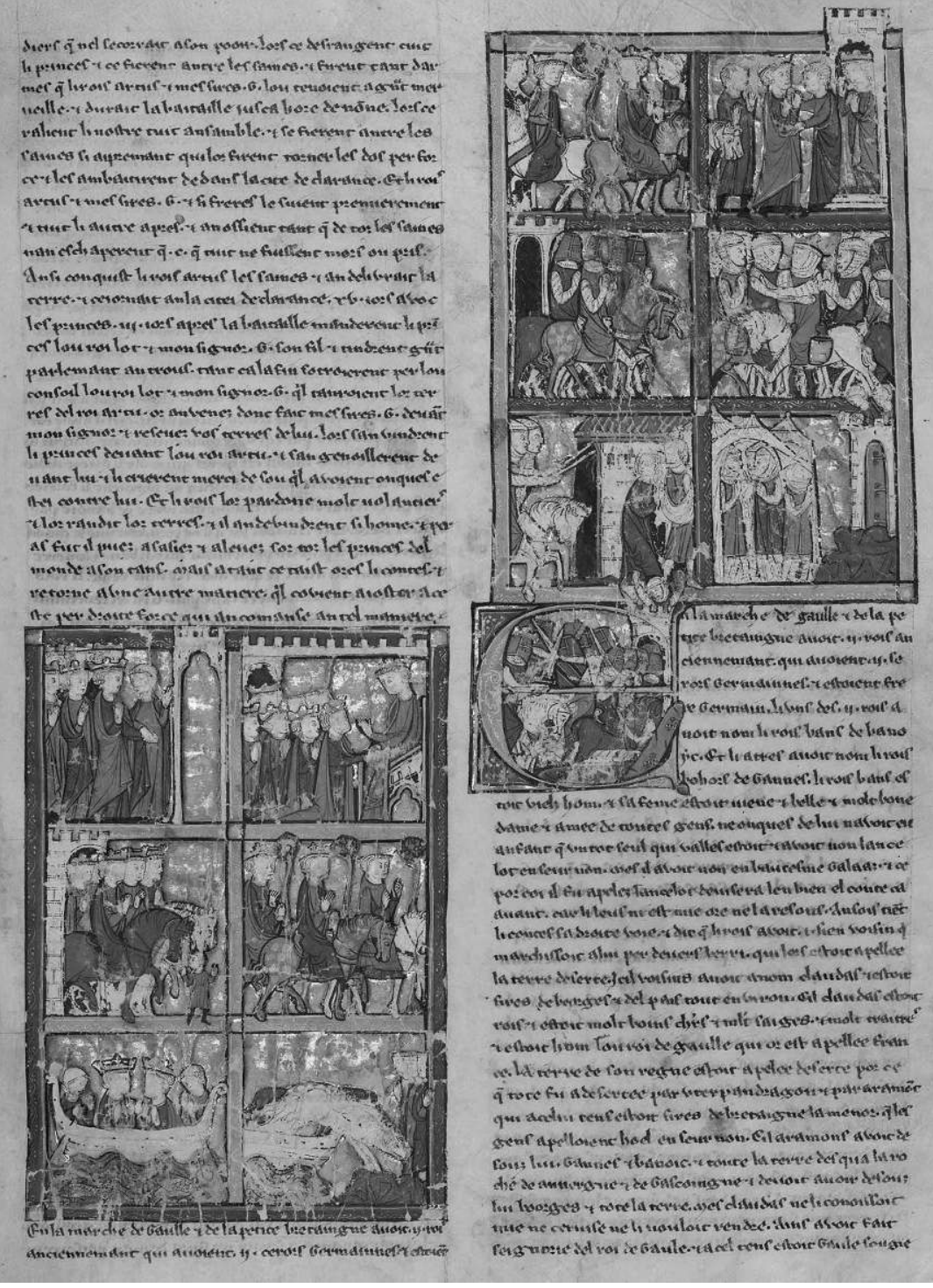

Figure 3. Paris, BnF, fr. 344, $\mathrm{f}^{\circ} 184$

Fin de la Suite Vulgate $\left(\mathrm{f}^{\mathrm{0}} 184 \mathrm{a}\right)$

Miniature:

Gauvain et les rois rebelles

Hommage des rois rebelles à Arthur

- Rois quittant Clarence

- Rois rentrant dans leurs pays

- Ban et Bohort rentrant dans leur pays

Débarquement de l'équipage de Ban et

Bohort
Début du Lancelot ( $\left.\mathrm{f}^{\mathrm{0}} 184 \mathrm{~b}\right)$

Miniature:

- Retour du roi Ban à Trèbes

- Sortie du sénéchal de Trèbes

- Trahison du sénéchal de Trèbes

- Incendie de la forteresse de Trèbes

- Siège de Trèbes

Initiale historiée:

Prise de Trèbes
- Fin des négociations entre Ban et Claudas 
Bohort se rendent à la cour d'Arthur à la demande de Merlin et y réitèrent leur hommage (Pl. I, p. 821). Malgré les troubles déjà causés par Claudas dans leur royaume, ils acceptent d'accompagner le jeune roi en Carmélide et en reçoivent la contrepartie quand Arthur leur prête main forte lors de la guerre de Gaule (Pl. I, p. 1168 sq.). À la fin de la continuation, dans le fr.344, l'arrivée dramatique des messagers des femmes de Ban et Bohort et l'urgence de la situation expliquent la rapidité de leur départ (BnF, fr. 344, $\left.\mathrm{f}^{\circ} 182 \mathrm{va}\right)$ :

Atant ez vos un mesaigier de part la roïne de Benoic. Et quant il vit lou roi Ban, si li escria et dist: «Hai, sire, que demourez vos ci? Jai vos mande ma dame la rö̈ne qu'elle ait de vos molt grant mestier, car li rois Claudes l'a assise et jure qu'il ne s'an partirait devant tant qu'il l'avrait prise. Et ce il puet tenir ma dame la roïne, elle cerait livree a honte, et vostre filz Lancelos cerait decopez piese a piese». Il n'ot mies conté sa parolle quant uns atres mesaiges antre leans de part la rö̈ne de Gaunes, la feme a roi Bohort, et quant il lou vit, si li escria: «Hai, sire, or poez trop longuemant si cejorner, car ma dame la roïne est an si grant peril et tote votre terre d'estre destruite a toz jors mais, que je ne cuit mies que vos i poïssiez jamais venir a tans. Car li rois Claudes vos art et destruit chascun jor vostre terre et si ait assise la roïne an Benoic.».

Le placement des deux dernières scènes de la miniature compartimentée, qui ne correspond pas à l'ordre du texte, pourrait s'expliquer par leur rôle de transition par rapport au début du Lancelot. La miniature frontispice du Lancelot (Figure 3), placée en diagonale de celle qui clôt la Suite Vulgate, montre l'échec des négociations entre Ban et Claudas et leur affrontement, ainsi que la trahison du sénéchal de Trèbes. Tandis qu'un premier conflit politique est réglé, c'est sur une autre aire géographique que se déplace l'attention. L'iconographie met en relation le départ des deux vassaux d'Arthur et leur retour dans leur pays avec le conflit qui oppose Ban de Bénoïc et Claudas de la Déserte, puisque Ban refuse de devenir le vassal de Claudas.

Le début du Lancelot rappelle l'histoire des royaumes de Gaunes et de Bénoïc et leur statut du point de vue féodal. Par le passé, Claudas a revendiqué le royaume de Bourges qui dépendait d'Arramont, le suzerain de Gaunes et de Bénoïc. Il a refusé de prêter hommage à Arramont et a choisi de se déclarer vassal du roi de Gaule, lui-même assujetti à Rome.

hiérarchie féodale (suzeraineté), et par surcrô̂t, tous les autres habitants du royaume, ceux des villes et ceux des campagnes (souveraineté) » (J.-F.LEMARIGNIER, La France médiévale..., p. 144 et 261). De fait, la reconnaissance de la suzeraineté du roi par ses grands vassaux est la plus difficile à obtenir. Au début de la Suite Vulgate, alors qu'Arthur est confronté à la rébellion de ses barons, il est immédiatement soutenu par le peuple et le clergé.
Devant affronter Claudas et ses alliés gaulois et romains, Arramont décide de prêter hommage à Uterpandragon en échange de son appui militaire. Après les événements narrés dans la Suite Vulgate, Claudas reprend à nouveau les armes contre Ban, par animosité envers Arthur, le suzerain de ce dernier. Claudas, qui assiège le roi Ban à Trèbes, lui propose de mettre fin aux hostilités à condition que Ban lui prête hommage, mais ce dernier refuse, par fidélité envers son suzerain ${ }^{24}$ :

Et li rois Claudas dist [au roi Ban]: «Je ne le vous toil mie pour chose que vous m'aiiés mesfait, ne pour haïne que j'aie a vous, mais pour ce que vous tenés le roi Artu pour signour. Et se vous voliés, je feroie biau plait a vous. Saisissés moi de cest chastelet je le vous renderai maintenant par tel covent que vous en devenrés mes hom, et tenras de par moi toute la toie terre». «Ce ne ferai je mie, ce dist li rois Bans, car je me parjuerroe envers le roi Artu, qui hom je sui liges $»^{25}$

La version remaniée du fr. 344 resserre le lien entre la fin de la Suite Vulgate et le début du Lancelot autour des relations féodales et plus particulièrement, des liens de vassalité qu'entretiennent Arthur et ses barons. La mise en perspective historique souligne les torts à la fois passés et présents de Claudas et ajoute à l'enjeu de la parole donnée l'idée d'une fidélité et d'un maintien du lien féodal qui s'inscrit dans le temps.

Le remanieur met en parallèle la question du rapport d'Arthur et de ses vassaux à la fin de la Suite Vulgate et les problèmes que pose le début du Lancelot. Dans le Lancelot, Claudas et Ban s'entendent finalement sur le fait que Ban demandera à Arthur de le secourir avant quarante jours, sans quoi il devra livrer sa terre à Claudas et lui rendre hommage. La trahison du sénéchal de Trèbes, qui livre la cité à Claudas, et la mort du roi Ban, parti en ambassade auprès d'Arthur, précipitent le destin des royaumes de Gaunes et de Bénoïc. Arthur ne vient pas au secours de ses vassaux et, dans

24. Dès les débuts de la société féodale (à la fin du IX $\mathrm{C}^{\mathrm{s}} \mathrm{s}$.), malgré l'effort pour promouvoir le caractère unique et absolu du lien vassalique, on constate des cas d'hommages multiples, ce qui constitue une des failles du système. À partir du $\mathrm{xl}^{\mathrm{e}}$ siècle, l'hommage lige tend à y remédier, mais il fait aussi rapidement l'objet d'une utilisation extensive. Voir M.B.ocH, "L'homme de plusieurs maîtres», dans La Société féodale. 1, La formation des liens de

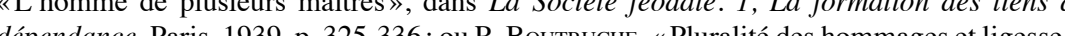
depens Seigneurie el féclalie, Pais, 1959, p. $162-2$ mages étaien cependant rendus pour des fiefs distincts, alors que Claudas prétend se substituer à Arthur comme suzerain de Ban pour le royaume de Bénoïc.

25. Le Livre du Graal, II. Lancelot, De «la Marche de Gaule» à «la Première partie de la quête de Lancelot», D. Poirion et Ph.WALTER dir., Paris, 2001, p. 10 [cité dorénavant $\mathrm{Pl}$. II]. La détermination de Ban contraste avec la trahison du sénéchal de Trèbes, qui livre à Claudas la forteresse contre la promesse d'en être réinvesti et de devenir son vassal (Pl. II, p. 17). 
le fr.344, le discours du messager de Bénoïc à la fin de la Suite Vulgate anticipe, au début du Lancelot, la plainte du religieux Adragain, qui se fait le porte-parole de la reine Élaine auprès du roi Arthur après le décès du roi Ban. Adragain reconnaît devant les reines Élaine et Évaine qu'Arthur a eu fort à faire dans son propre royaume (Pl. II, p. 95) :

«Et non pour quant je sai bien que li rois Artus a eü tant a faire que ce n'est mie grant merveille qu'il a ceste chose tant mise en delai, car il n'i a gaires baron en sa terre qu'il ne li ait menee guerre, tant que maintes gens ont quidié qu'il en remansist essiliés en le pardefin. »

Les démêlés d'Arthur et de ses barons constituent ainsi la matière principale de la fin de la Suite Vulgate dans la version abrégée transmise dans le fr.344. Le religieux expose publiquement la faute commise par Arthur à l'égard de ses hommes liges, Ban et Bohort (Pl. II, p. 96-97):

«Il est voirs que tu es li rois el monde orendroit, et de coi on ait oï parole, qui plus maintient chevalerie en grant honour; et plus fais grans biens que nuls rois dont on ait parlé jusques ci, selonc Dieu et selonc le monde. Mais trop es pereçous de vengier les hontes et les damages que on te fait: car qui fait honte et damage a ton home, on le fait a toi; et quelconques damage c'on face a ton home, la honte en est toie. Tu honneures et doutes et sers ciaus qui desloiaument te guerroient et courent sus, et ciaus oublies et més ariere qui t'ont loiaument servi sans fausser; et ont perdu et terres et honours et lor vies, et sont en aventure de lor ames par ton service.»

La question féodale des relations entre souverain et vassaux est donc centrale aussi bien dans la suite du Merlin que dans le Lancelot. Mais, par contraste avec les autres manuscrits de la Suite Vulgate, le remaniement de la fin du texte dans le fr.344 a pour conséquence la suppression du passage final consacré à la naissance de Lancelot, Lionel et Bohort (Pl. I, p. 16601662), et introduit de façon différente le conflit qui oppose Ban et Bohort à Claudas ${ }^{26}$. La fin abrégée du fr.344 avance le retour de Ban et de Bohort dans leur pays. Les deux rois, qui ont un temps suppléé aux barons révoltés contre Arthur, ont bénéficié de son aide au cours de la guerre de Gaule. Le remaniement joue sur la temporalité de la fin de la continuation et du début du Lancelot. Ban et Bohort sont livrés à eux-mêmes au moment précis où leur suzerain se concentre sur la protection de l'intégrité du royaume breton

26. Ce passage joue un rôle de transition et d'annonce du Lancelot, mais il présente aussi une discordance du point de vue de la construction cyclique, à travers la mention problématique de la mort du sénéchal Pharien qui fait l'objet de diverses réécritures dans la version courte de la Suite Vulgate. Voir R.TRACHSLER, «Pour une nouvelle édition de la SuiteVulgate du Merlin»..., p. 128-148. contre les Saxons. La suppression de la fin de l'Estoire de Merlin ne résout pas l'écart temporel qui sépare ce texte du début du Lancelot ${ }^{27}$. Cependant, la version condensée adoptée par le fr. 344 justifie partiellement la défaillance d'Arthur à l'égard de ses vassaux, car elle semble faire de l'attaque de Claudas et de la coalition des Chrétiens contre les Saxons des événement concomitants. Le roi déroge à son devoir de suzerain, non pas en raison de sa faiblesse et de son apathie, mais à cause d'autres engagements militaires.

\section{Transition cyclique et exaltation de la souveraineté d'Arthur}

Dans le fr.344, la dernière phrase de la Suite Vulgate fait l'éloge de la suprématie à laquelle parvient Arthur, au paroxysme de son règne, «asasiez et alevez sur toz les princes del monde a son tans» ( $\left.f^{\circ} 184\right)$. Elle accentue l'effet de clôture des Premiers Faits d'Arthur, puisque désormais est reconnue l'autorité d'un souverain dont la légitimité était remise en cause au début de la continuation ${ }^{28}$. Cette conclusion souligne le rayonnement international du roi de Grande Bretagne, alors qu'Arthur affirme d'abord sa souveraineté parmi ses vassaux les plus proches et sur le territoire breton. L'abrègement du fr. 344 conduit en effet à l'omission de la guerre contre les Romains, opérant un recentrement sur le domaine anglais et les territoires environnants. Le passage de la lutte contre Rome, construit en symétrie inversée par rapport à la Mort Artu, peut sembler redondant dans une perspective cyclique, puisqu'il est tiré d'un seul et même épisode chez Geoffroy de Monmouth ${ }^{29}$.

Arthur est exalté, moins pour sa prouesse individuelle que dans sa stature politique et militaire, comme l'indique l'absence du duel entre Arthur et Rion, de l'aventure du Géant du Mont Saint-Michel et du combat contre le chat de Lausanne, des épisodes hérités de l'Historia Regum Britanniae de Geoffroy de Monmouth et du Brut de Wace, qui exaltent l'image mythique

27. Sur l'entrée in medias res de ce texte, voir A.Combes, «Le prologue en blanc du Lancelot en prose», dans E.BAUMgarTner et L.HARF-LANCNER éd., Seuils de l'œuvre dans le texte médiéval, Paris, 2002, p. 21-52.

28. À l'origine, le pouvoir d'Arthur ne provient pas du système féodal, puisque les grands barons rejettent sa suzeraineté, mais de sa foi en Dieu et du fait qu' «avant même d'avoir été sacré roi, il s'en remet au haut clergé pour les décisions importantes». On peut y voir la trace de l'augustinisme politique dont les théoriciens unt de la religion chétiente voir la trace de l'a à d'autres systemes idéologiques et politiques: féodalité, système des trois ordres, lutte d'influence entre le pouvoir royal et la papauté. Voir D. Boutet, Charlemagne et Arthur ou le Roi imaginaire..., p. 169-71 et 184-85; H.-X.ARQUILlière, L'Augustinisme politique : essai sur la formation des théories politiques du Moyen Age. Paris, 1934, p. 68-104.

29. GeOFFREY OF MonMouth, The History of the Kings of Britain. An Edition and Translation of De gestis Britonum [Historia Regum Britanniae], éd. M. REevE, trad. N. WRIGHT, Woodbridge, 2007, § 158-76. 
du souverain. La souveraineté d'Arthur, chef de guerre et roi légitime, ancré dans un récit certes romanesque mais à prétention historique, importe davantage que son prestige de héros civilisateur. La conclusion proposée par le fr.344 se concentre sur le rétablissement de l'ordre au sein du royaume breton. La question de l'unité du royaume et de son bon gouvernement sert alors de transition entre la Suite Vulgate et le Lancelot.

L'exaltation d'Arthur et de la figure du souverain, qui présente la continuation du Merlin comme «une somme au profit de la royauté ${ }^{30}$ », fait ressortir la perspective idéologique propre à la Suite Vulgate au sein du cycle du Graal, tout en annonçant certains enjeux introduits au début du Lancelot. Si ce dernier texte remet en question la capacité d'Arthur à assumer ses devoirs de suzerain, il le présente toujours, du moins en son commencement, comme un souverain modèle ${ }^{31}$. Claudas lui-même le reconnaît, quand il se rend incognito à la cour de son adversaire ${ }^{32}$. Les qualités d'Arthur et l'attraction que suscite sa cour sont immédiatement associées à des enjeux d'ordre vassalique. En effet, l'écuyer qui a accompagné Claudas à la cour d'Arthur reconnaît que si son seigneur décidait d'attaquer Arthur, il devrait prendre la défense de ce dernier (Pl. II, p. 65):

«Conment! dist Claudas, si li aideriés contre moi qui vos liges sires sui, et t'ai fai riche et honeré pour ton service! [...] Dont seroie-tu, fait Claudas, desloiaus et traïtres envers moi qui tes sires sui, si com tu meïsmes le connois, car tu es mes hom liges et tu seroies en l'aïde d'un estrange home contre moi. »

L'écuyer se justifie de toute accusation de traitrise en précisant qu'il romprait auparavant son hommage envers Claudas, et qu'en prenant le parti d'Arthur, le parangon de la chevalerie, il œuvrerait pour le bien du monde entier. Des exigences morales supérieures cautionneraient ainsi la rupture du lien vassalique et la mise en place d'une nouvelle allégeance fondée sur le mérite exceptionnel d'Arthur, dont les qualités justifient pleinement une suzeraineté et une souveraineté élargies.

30. D. Boutet, Charlemagne et Artur ou le Roi imaginaire..., p. 41-43. Au moment où s'achève «l'intégration de l'idée de chevalerie dans l'idéologie royale», Arthur est présenté la Suite Vulgate comme le phare de la chevalerie, alors que, dans la Queste, il est «exclu de l'aventure suprême».

31. Sur la représentation d'Arthur, en tension avec les figures de Claudas et de Galehaut dans le Lancelot en prose, voir E. KenNedy, «King Arthur in the First Part of the Prose Lancelot», dans F.Whitehead, A. H.Diverres et F. E.Sutcliffe éd., Medieval Miscellany presented to Eugène Vinaver by Pupils, Colleagues and Friends, Manchester, 1965, p. 18695; ID., «Études sur le Lancelot en prose», Romania, 105 (1984), p. 46-62.

32. Claudas admire alors «sa largece et sa debonaireté et son grant sens et sa bonté: si le vit de toutes valours si plain et si entechié de cuer et de cors qu'il ne prisoit envers lui nul home dont il eüst parole oïe» (Pl. II, 62).
La formule qui clôt la Suite Vulgate dans le fr.344 (fo 184va), «Mais atant ce taist ores li contes et retorne a une autre matiere qu'il covient ajoster a ceste per droite force, qui ancomanse en tel maniere», joue de l'entrelacement pour opérer la transition entre la suite du Merlin et le Lancelot. Elle constitue une variation sur l'expression adoptée à la fin de l'Estoiredel saint Graal $^{33}$. La phrase de transition utilisée à la fin de la Suite Vulgate ne reprend pas l'image de la branche ${ }^{34}$ mais souligne, comme celle qui lui sert de modèle, l'effort qui consiste à assembler des ensembles narratifs distincts. Le Lancelot est alors présenté comme une «autre matière», une expression qui rappelle son altérité à l'égard du Merlin et de sa continuation. L'utilisation de cette formule peut apparaître comme une façon de préserver l'indépendance des différentes parties du cycle et d'en expliquer les possibles écarts et contradictions. L'exaltation de la figure d'Arthur, dont les failles seront certes explorées au cours du Lancelot, sert pourtant de trait d'union entre ces œuvres. Le remaniemen textuel et la mise en page de la fin de la continuation contribuent en effet à l'intégration de la Suite Vulgate et du Lancelot par leur focalisation sur des enjeux politiques et féodaux.

Le programme iconographique de plusieurs manuscrits de la Suite Vulgate se termine sur la représentation du retour de Ban et Bohort dans leur pays ou sur la mise en scène de leurs enfants. Les images insistent souvent à l'instar du récit, sur la quasi-gémellité des couples royaux et la symétrie harmonieuse des familles de Ban et Bohort ${ }^{35}$. La stratégie visuelle adoptée dans l'illustration du fr.344 fait ressortir le seuil des œuvres qui constituen le cycle du Graal, en insistant moins sur leur autonomie que sur la formation d'un ensemble cohérent dont les articulations sont particulièrement soignées De même que la fin traditionnelle de la continuation, le texte et la miniature de la fin de l'Estoirede Merlin dans le fr.344 jouent un rôle d'ouverture cyclique et de transition par rapport au Lancelot copié à sa suite, puisqu'ils opèrent un déplacement spatial et se focalisent sur les personnages qui seront mis en scène dans l'œuvre qui suit. La succession lignagère inscrit le passage de la Suite Vulgate au Lancelot dans la perspective d'une continuité historique et généalogique. Dans certaines miniatures, comme dans Paris, BnF, fr. 95 , f 354 (Figure 5), l'absence de Ban et de Bohort anticipe peut-

33. « Si se test ore li contes atant de totes les ligniees qui de Celidoine issirent et retorne a une autre branche que l'en apelle l'estoire de Merlin qu'il convient ajoster ensemble a fine force avec l'estoire del saint Graal, por ce que branche en est et apartient» (Paris, BnF, fr. 344, fo 81 ).

34. Sur l'utilisation narrative et poétique de la métaphore de la greffe, voir F.GingRAs, «De branche en branche: aux racines des coupes romanesques», dans V.FASSEUR, D. JAMESRaOul et J-R ValetTe éd., L'Arbre au Moyen Âge, Paris, 2010, p. 183-196.

35. Voir Bonn, ULB, 526; Paris BnF, fr. 19162 (Figure 4), et fr.24394, qui datent de la seconde moitié du XIII siècle; ou New Haven, Beinecke Library, 227 (1357). 
être la disparition précoce de ces souverains et le désordre politique qui en résulte au début du Lancelot. Sur le plan textuel et visuel, ces manuscrits soulignent la façon dont la fin de la Suite Vulgate prépare le début du Lancelot et assume à son égard une fonction de transition.

\section{retorne apt aet ran van a tenghe ec ael ivi toljo:e fon fíe tirione en los tives}

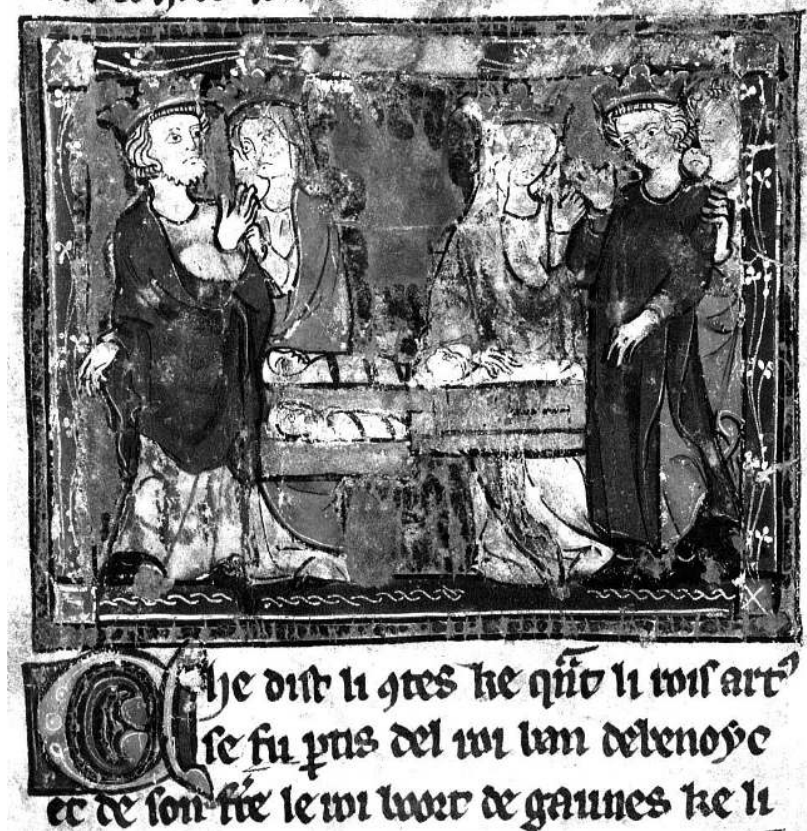

Figure 4. Fin de la Suite Vulgate: Ban et Bohort et leur famille. Paris, BnF, fr. $19162, \mathrm{f}^{\circ} 372$

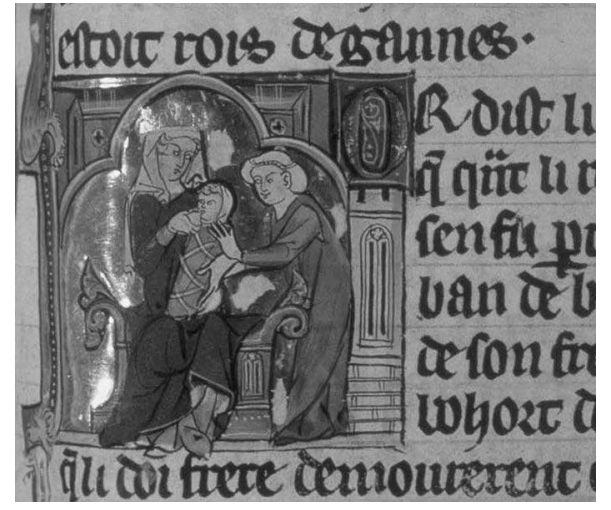

Figure 5. Fin de la Suite Vulgate: Elaine et Lancelot. Paris, BnF, fr.95, $\mathrm{f}^{\circ} 354$.
La miniature finale de la Suite Vulgate dans le fr.344 se situe dans la continuité des illustrations qui l'ont précédée et montre combien la relation entre le roi et ses vassaux constitue un enjeu primordial du Merlin et de sa continuation. Une perspective similaire semble animer le remanieur de la fin du texte. D'un côté, l'usurpateur Vertigier met à mort les assassins du roi Maine, comme pour renier le crime qui rend possible son accès au trône de Bretagne. De l'autre, Arthur, dont la légitimité était d'abord contestée parvient à rallier à sa cause les rois révoltés. Le programme iconographique, qui privilégie la Suite Vulgate sur le Merlin propre, souligne les enjeux politiques et militaires de la continuation, et met en valeur les épisodes remaniés. Le recours à deux miniatures compartimentées à la fin de la Suite Vulgate et au début du Lancelot insiste visuellement sur l'articulation de ces deux textes dans une perspective cyclique.

Le fr.344 se termine sur le bilan des progrès militaires du jeune souverain, plutôt que sur la figure de Merlin. L'abrègement de la fin du texte qui se fait au détriment d'épisodes romanesques, permet de se concentrer sur l'affirmation guerrière de l'autorité d'Arthur au sein de son royaume. La question des relations féodales et du rapport entre le souverain et ses vassaux, également explorée dans les cycles épiques, sert de fil directeur entre la fin de la continuation et le début du Lancelot. Dans la version du cycle du Graal proposée par le fr. 344, la concentration et la redistribution de la matière narrative relativement hétérogène de la Suite Vulgate permettent de cristalliser la nature historico-épique de ce texte. Cela laisse aux autres œuvres du cycle l'exploration des aventures des chevaliers arthuriens et la déconstruction de la figure du souverain. L'abrègement et le remaniement de la fin de la Suite Vulgate dans le fr.344 ne contribuent pas de façon univoque à un renforcement du caractère cyclique de l'œuvre. À la fin du Moyen Âge, plusieurs textes dont le De casibus virum illustrium de Boccace et les différentes versions des Neuf Preux «essaient de recentrer la matière arthurienne sur son personnage original et de rapporter donc in extenso la biographie d'Arthur ${ }^{36} \gg$. Dans le cycle du Graal, la Suite Vulgate redessine l'histoire d'Arthur en insistant sur la jeunesse guerrière du roi de Bretagne, mais aussi sur les questions complexes des relations féodales et du statut de la figure royale, un aspect renforcé dans la version remaniée que donne de la fin du texte le fr. 344

On ne connaît pas le commanditaire ou les premiers possesseurs du fr.344 à la toute fin du XIII ${ }^{\mathrm{e}}$ siècle $^{37}$. Cependant, le dialecte du texte

36. R. Trachsler, Clôtures du Cycle Arthurien. Étude et Textes, Genève, 1996, p. 356. 37. Plusieurs marques d'appartenance indiquent qu'il a ensuite été en possession de Marie de Hainaut (1280-1354), fille de Jean II d'Avesnes, comte de Hainaut, après son mariage avec Louis I, duc de Bourbon en 1310. Voir l'ex libris $\mathrm{f}^{\circ} 1$ : «Marie de Hainaut, duchesse de Bo [urbon]» répété avec un code consistant à remplacer les voyelles par d'autres lettres de l'alphabet «Mbrkf df hbknbxt dxchfssf df bp ...» (R. MIDDLETON, «The Manuscripts», dan 
et l'examen des miniatures suggèrent que le manuscrit a été produit en Lorraine, aux alentours de Metz ou Verdun, des évêchés ayant le statut de villes libres du Saint-Empire Romain Germanique qui comme le comté de Bar sont situés entre des terres d'allégeance germanique (le duché de Lorraine et le comté de Luxembourg), ou d'appartenance française (le comté de Champagne $)^{38}$. Le remanieur témoigne d'un intérêt particulier pour les conflits d'allégeance qui peuvent se poser dans le monde féodal, notamment en ce qui concerne des territoires placés entre différentes zones d'influence. Or, les questions féodales et la relation entre le roi et ses vassaux trouvent aussi un terrain d'expression privilégié dans la chanson de geste, comme dans le cycle de Doon de Mayence, également appelé cycle des barons révoltés ${ }^{39}$. On peut mettre en relation la production du fr. 344, un manuscrit cyclique arthurien remanié, avec une littérature épique à caractère régional destinée au même public ${ }^{40}$. Le cycle des Lorrains, qui comprend cinq chansons de geste composées entre la fin du XII et le début du XIII ${ }^{\mathrm{e}}$ siècle ${ }^{41}$, exhibe ainsi les implications de la solidarité lignagère et interroge le rôle de l'autorité royale dans la société féodale, tout en conservant en arrièreplan le thème de la lutte contre les Sarrasins. Les remaniements et les trois mises en prose ultérieures de ce cycle épique témoignent de son succès et privilégient le noyau formé par Garin le Lorrain et Gerbert de Metz. Les héros sont habituellement des seigneurs pris dans des querelles lignagères ou en conflit avec l'autorité royale. Cependant, la Vengeance Fromondin ${ }^{42}$, qui constitue une conclusion possible mais problématique de la geste des Lorrains, se place dans une perspective idéologique qui va à l'encontre de celle développée dans le reste de ce cycle. Elle fait la promotion du roi de France, Pépin, au détriment des seigneurs du Nord: du temps de Philippe Auguste, «le poète utilise les personnages de la fiction pour servir son

G. S. BuRgess et K. PratT éd., The Arthur of the French: The Arthurian Legend in Medieval French and Occitan Literature, Cardiff, 2006, p. 62).

38. Voir Ch. Aimond, Les Relations de la France et du Verdunois de 1270 à 1552, Paris, 1910 ; J. SchNeIDER, La Ville de Metz aux XIII' et XIVe siècles, Nancy, 1950; M. PARISSE éd., Histoire de la Lorraine, Toulouse, 1977, p. 153-199; A. GiRARDOT éd., Histoire de Verdun Toulouse, 1982, p. 77-198 ; F.-Y.Le MolGNe éd., Histoire de Metz, Toulouse, 1986, p. 137 -

39. Alexandre Micha notait déjà que l'opposition des grands vassaux, qui peut s'expliquer par la naissance trouble d'Arthur, manifeste également l'influence de la production épique qui par la oppose à Charlemagne des figures prestigieuses comme Girart de Vienne, Girart de Roussillon Ogier le Danois, et présente le danger qui menace le jeune Louis dans le Couronnement. A. Micha, «Les sources de la Vulgate du Merlin», dans De la chanson de geste au roman, Genève, 1976, p. 301

40. Je remercie Catherine Leglu pour cette suggestion.

41. Voir notamment Hervis de Mes, chanson de geste anonyme (début du XIII'siècle), ed. J.-C. Herbin, Genève, 1992; Garin le Loherenc, éd. A. Iker-Gittleman, Paris, 19961997; Gerbert de Mez: chanson de geste du XII siècle, éd. P. TAYLOR, Namur, 1952.

42. La Vengeance Fromondin éd. J.-C. HerBin, Paris, 2005. dessein politique et publicitaire au profit de la monarchie capétienne ${ }^{43} »$. La Suite Vulgate du Merlin et le remaniement du fr.344 abordent les mêmes questions politiques et féodales dans un contexte arthurien et breton.

Irène FABRY-TEHRANCHI - University of Reading

Arthur et ses barons rebelles. La fin remaniée et abrégée de la Suite Vulgate du Merlin dans le manuscrit du cycle du Graal (Paris, BnF, fr. 344, ca 1295)

Le ms. Iorrain Paris, BnF, fr. 344, datant de la fin du XIII' siècle, conserve l'intégralité du cycle du Graal et présente une version abrégée de la fin de la Suite Vulgate du Merlin. Le remaniement de la fin de la continuation met entre parenthèses les épisodes plus romanesques de la version commune et construit la cohérence de l'œuvre autour de la figure d'Arthur, souverain légitime et habile chef de guerre, confronté à la dissidence de ses barons. De la fin du fo 182 au fo $184 v^{\circ}$ sont évoqués le départ des rois Ban et Bohort pour le continent, l'ambassade du roi Loth et de ses fils, et la lutte contre les Saxons de la coalition chrétienne rassemblée à Logres pour la fête de la sainte Croix. Cet article montre comment l'abrègement et l'illustration de la fin du texte dans le fr. 344 témoignent d'une forte inflexion idéologique, exaltant la souveraineté d'Arthur en insistant sur le ralliement puis la contrition et la soumission de ses vassaux révoltés.

manuscrit - Suite Vulgate - Arthur - souveraineté - vassaux - cycle du Lancelot-Graal

Arthur and his Rebel Barons. The Abridgment and Remaniement of the End of the Vulgate Sequel to Merlin in the Lancelot-Grail Manuscript (Paris, BnF, fr. 344, ca 1295)

The ms. Paris, BnF, fr. 344 was produced in Lorraine at the end of the thirteenth century and contains the whole Lancelot-Grail cycle. It presents an abridged version of the end of the Vulgate Sequel to Merlin. The rewriting of the end of the sequel glosses over the romantic episodes of the common version and focuses on the figure of Arthur, a legitimate sovereign and skilful war leader confronted by his barons'dissidence. From the end of $f^{\circ} 182$ to $f^{\circ} 184 v^{\circ}, B n F$, fr. 344 , narrates the departure of Kings Ban and Bohort for the Continent, the embassy of King Loth and his sons, and the fight against the Saxons of the Christian coalition gathered at Logres for the feast of the Holy Cross. This article shows the ideological implications of the abridgement and the illustration used at the end of the Vulgate Sequel in ms. fr. 344, as it exalts Arthur's kingship and insists on the rallying, penance and submission of his rebelled vassals.

manuscript - Vulgate Sequel - Arthur - kingship - vassals - Lancelot-Grail cycle

43. J.-C. Herbin, «Variations, vie et mort des Loherains», Cahiers de recherches médiévales, 12 (2005), p. 147-174. 
